\title{
DOS ESPECIES DE AMBROSIA (COMPOSITAE, HELIANTHEAE) ADVENTICIAS EN EL CENTRO DE MEXICO
}

\author{
JERZY RZEDOWSKI ${ }^{1}$ \\ Y \\ Graciela Calderon de Rzedowski \\ Instituto de Ecología, A.C. \\ Centro Regional del Bajío \\ Apartado postal 386 \\ 61600 Pátzcuaro, Michoacán
}

\begin{abstract}
RESUMEN
Ambrosia camphorata (Greene) Payne y A. cordifolia (A. Gray) Payne muestran una distribución notablemente discontinua, pues por una parte se registran de amplios sectores de la zona árida sonorense y por la otra prosperan en una porción relativamente limitada de San Luis Potosí, Guanajuato y Querétaro. En virtud del hecho de que el centro de diversidad y de probable origen de este grupo de plantas se encuentra en el noroeste de México, así como de la circunstancia de que las dos plantas dan la impresión de comportarse como invasoras en el centro del país, se piensa que ambas deben haber llegado a esta última región en tiempos relativamente recientes y se postula el siglo XIX como la posible época del movimiento migratorio.
\end{abstract}

\section{ABSTRACT}

Ambrosia camphorata (Greene) Payne and A. cordifolia (A. Gray) Payne reveal a remarkable disjunct distribution. Both are members of the flora of large areas of the Sonoran arid zone and also grow in a relatively limited portion of the states of San Luis Potosí, Guanajuato and Querétaro. In view of the fact that the center of diversity and of probable origin of this group of plants is localized in northwestern Mexico and on account of their apparent behavior as invasors in the central part of the country, it is believed that these species should have arrived in the latter region in relatively recent times. The XIX century is postulated as a possible epoch of their migratory movement.

En el transcurso de recorridos realizados en la zona limítrofe entre los estados de Guanajuato, Querétaro y San Luis Potosí ha llamado la atención de los que escriben la presencia de dos elementos de la familia Compositae.

Ambos son componentes importantes de la flora de la zona árida sonorense y es factible que su llegada al centro de México se ha realizado en tiempos no muy remotos, con toda probabilidad gracias a la intervención del hombre. A tal conclusión, al menos,

${ }_{1}$ Trabajo realizado con apoyo económico del Instituto de Ecología, A.C. (cuenta 902-03), del Consejo Nacional de Ciencia y Tecnología y de la Comisión Nacional para el Conocimiento y Uso de la Biodiversidad. 
Acta Botánica Mexicana (1998), 43:57-66

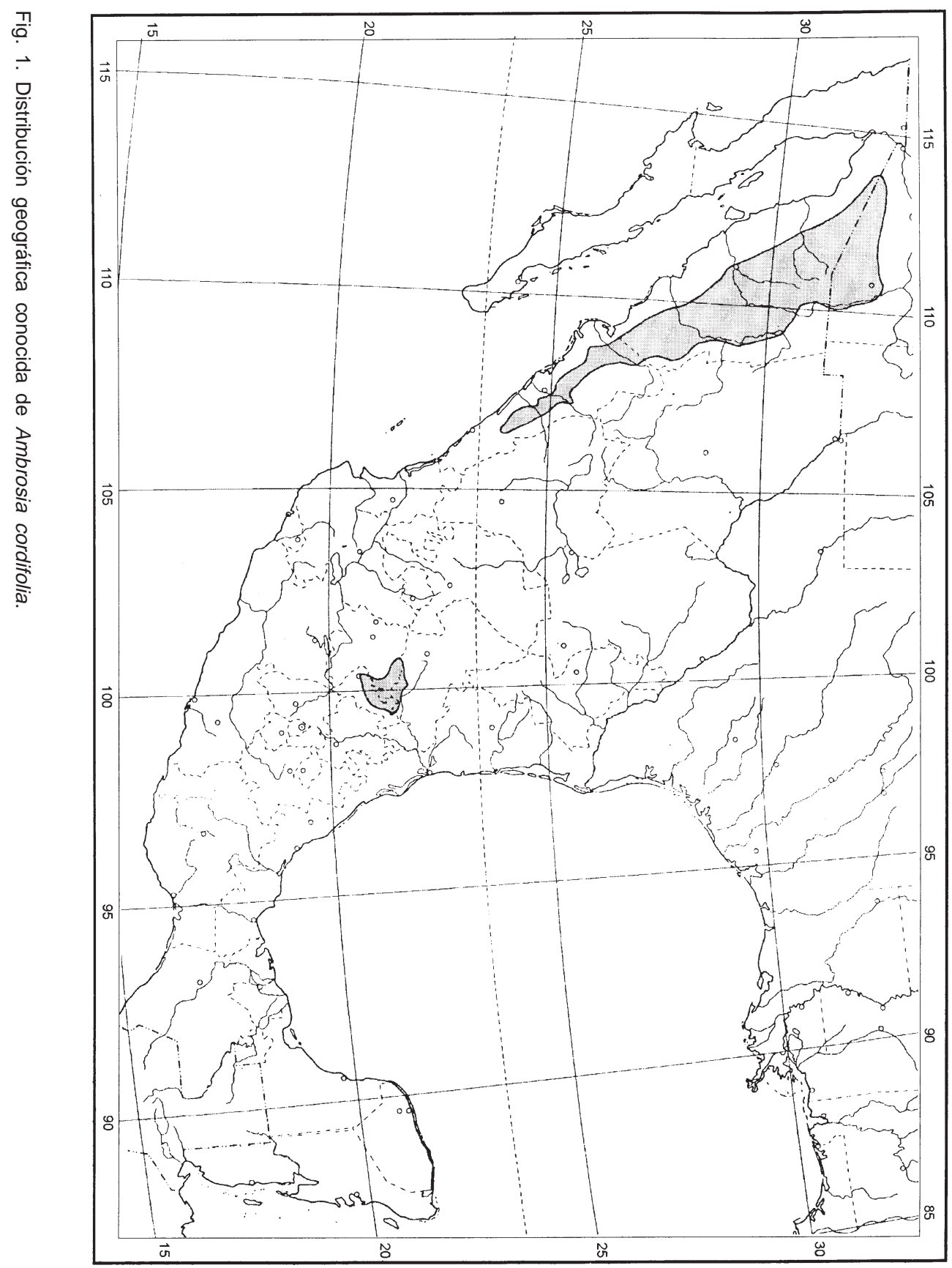


Rzedowski y G. Calderón de Rzedowski: Dos Especies Adventicias de Ambrosia

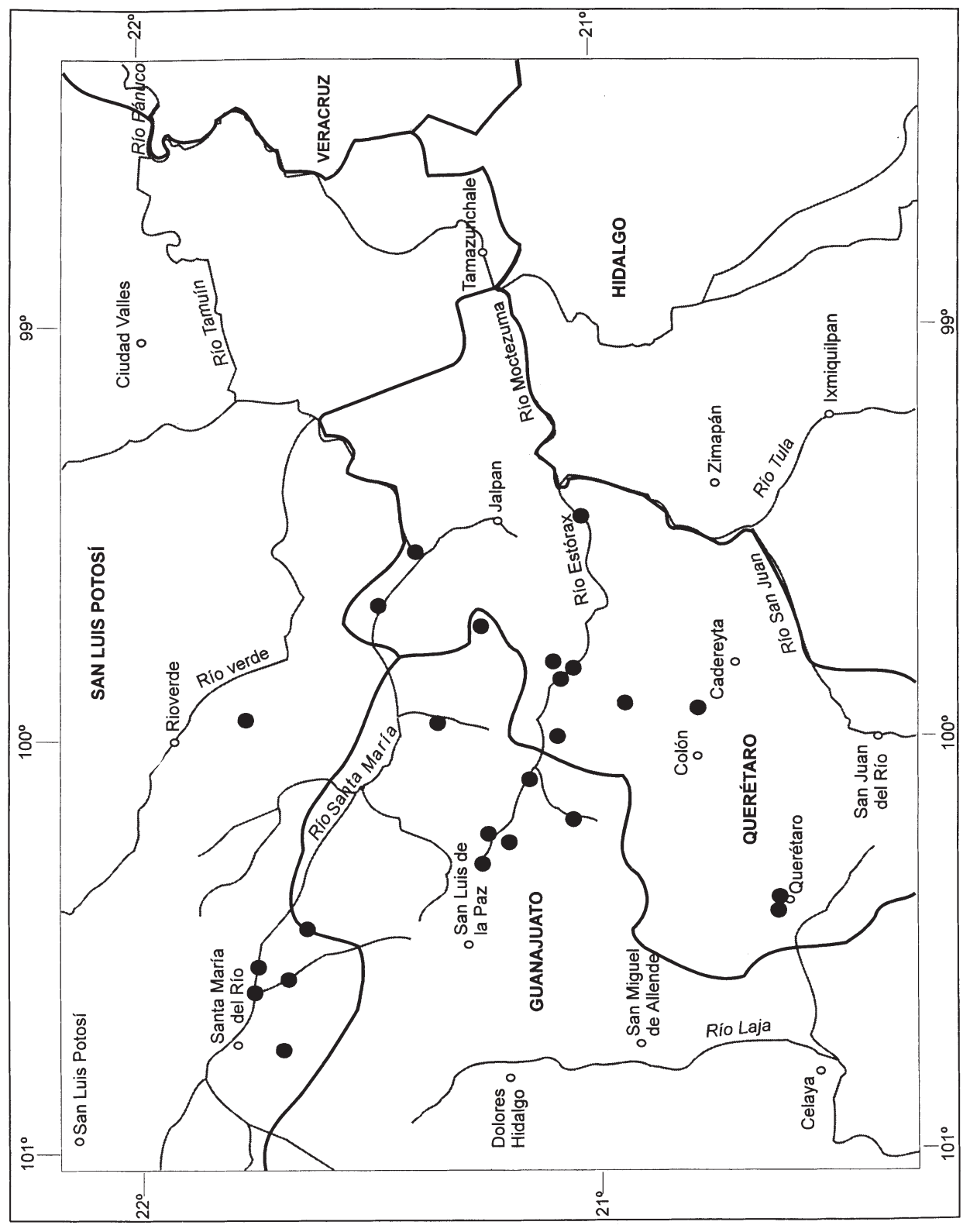

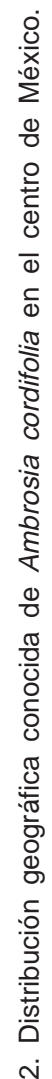
iำ 
conduce la distribución discontinua de las dos, unida al hecho de que dan la impresión de comportarse como invasoras en esta parte del país.

Aunque no muy cercanamente emparentadas entre sí, tanto una como la otra, se han descrito originalmente como miembros del género Franseria, caracterizado por sus propágulos espinudos ("abrojos"), que funcionan como ectozoocoras y pueden dispersarse con ayuda de animales, incluyendo al hombre mismo. Payne (1964), después de estudiar todo el conjunto, llegó a la conclusión que Franseria no puede mantenerse como entidad independiente de Ambrosia y en consecuencia transfirió allí a todas las especies de la primera.

A continuación se resumen las observaciones realizadas, así como otro tipo de información reunida para cada una de estas plantas.

Ambrosia cordifolia (A. Gray) Payne (=Franseria cordifolia A. Gray) es un arbusto de 0.7 a 1.5(2) m de alto, que se dio a conocer en 1884 del sur de Arizona. Rydberg (1922) registró su presencia en ese último estado, así como en Sonora y Sinaloa, y describió además como nueva a F. malvacea, con el tipo procedente del centro de Sinaloa y cuya distribución conocida se extendía a Sonora y a San Luis Potosí. Blake (1926) redujo a F. malvacea a la sinonimia de $F$. cordifolia y confirmó su existencia en los cuatro estados mencionados.

Gentry (1942) la definió como componente cuantitativamente muy significativo del bosque espinoso y del bosque tropical caducifolio que prosperan sobre laderas basálticas del sur de Sonora. Shreve (1951) puso de manifiesto la importancia de la especie en la composición de los matorrales xerófilos de varios sectores del mismo estado.

Zamudio Ruiz (1984) citó a A. cordifolia como participante de la vegetación de la zona árida del centro de Querétaro, mientras que Rzedowski et al. (1996) hicieron constar su presencia en la flora del NE de Guanajuato.

El mapa de la Fig. 1 sintetiza la distribución geográfica hasta ahora conocida de la especie en cuestión. Se basa en buena parte en la cartografía de Hastings et al. (1972) para la zona árida sonorense, así como en materiales existentes en los herbarios mexicanos para el resto de su área.

El mapa de la Fig. 2 detalla las localidades conocidas en el centro de la República. De acuerdo con la información contenida en este último, el arbusto llega a ocupar una superficie importante a lo largo de las cuencas de los ríos Santa María y Estórax, además de un par de sitios cercanos a la ciudad de Querétaro. Recibe allí los nombres comunes de "amargosa", "rama amarga" y "vara de cuete". Su amplitud altitudinal registrada en esta zona es de 500 a $2000 \mathrm{~m}$ y aunque el habitat general preferencial lo constituyen los matorrales xerófilos, marginalmente también penetra hacia terrenos en que prevalecen el bosque tropical caducifolio y el encinar. En forma definitiva prefiere suelos derivados de roca volcánica (riolita y basalto) y solamente de manera excepcional se ha observado sobre calizas o lutitas.

Una serie de observaciones cuidadosas revela, sin embargo, que en San Luis Potosí, Guanajuato y Querétaro $A$. cordifolia sólo forma parte de matorrales y de bosques con alto grado de disturbio, prosperando en comunidades modificadas o secundarias y con más frecuencia a orillas de veredas, caminos, vías de drenaje y en otros ambientes inestables, donde a menudo constituye masas densas de cientos o miles de individuos. 
Ambrosia camphorata (Greene) Payne (=Franseria camphorata Greene) es un arbusto hasta de $30(40) \mathrm{cm}$ de alto, sumamente aromático al estrujarse, que se describió en 1885 a base de ejemplares procedentes de la Isla Guadalupe y de la Isla Cedros, B. C.; Blake (1926) la registró de Baja California y de Sonora.

Según Shreve (1951), la planta se distribuye a lo largo de una extensa porción de la Península y llega a prevalecer en matorrales xerófilos que prosperan sobre suelos rojos derivados de roca basáltica en la región de Vizcaíno. Moran (1996) la señala como dominante en varios sectores de la parte meridional de la Isla Guadalupe.

Payne (1964) fue el primero en dar formalmente a conocer la existencia de la especie en el sur de San Luis Potosí, de donde también la había registrado Rzedowski (1961) bajo el nombre de Franseria sp.

De acuerdo con los datos revisados, $A$. camphorata no se había citado en la literatura del estado de Guanajuato, en el cual aparentemente no se le ha encontrado sino hasta hace relativamente poco.

El mapa de la Fig. 3 muestra a grandes rasgos el área total de la distribución conocida de esta especie. Para su elaboración, al igual que en el caso anterior, se partió de la carta correspondiente del trabajo de Hastings et al. (1972) para la zona árida sonorense, así como de la información contenida en los principales herbarios mexicanos.

En la Fig. 4 se señalan el área y las localidades del centro de México en las que se le ha encontrado y/o colectado.

Como puede observarse en estas cartas, la repartición de $A$. camphorata al sur del Trópico de Cáncer se limita a una extensión relativamente pequeña que se ubica a lo largo del cauce superior del Río Santa María, de algunos de sus afluentes, así como de escasas áreas circunvecinas. En la comarca la planta se conoce con el nombre común de "mariola". Las colectas en existencia proceden de los municipios de San Felipe, San Diego de la Unión y San Luis de la Paz del lado de Guanajuato. Las correspondientes a San Luis Potosí provienen de los municipios de Santa María del Río, Tierra Nueva y Villa de Reyes. La zona se caracteriza por extensos afloramientos de rocas volcánicas del tipo de las riolitas, en las cuales el sistema de drenaje ha excavado valles someros a medianamente profundos, cuyas altitudes oscilan entre 1750 y 2050 m de altitud.

De acuerdo con las observaciones realizadas en fechas recientes, en todos los sitios de San Luis Potosí y de Guanajuato en que se ha visto $A$. camphorata, dicha planta es muy abundante, con densidades que oscilan entre 1 a 3 individuos por metro cuadrado, excluyendo a menudo por completo o casi por completo a otros componentes arbustivos de talla inferior a $1 \mathrm{~m}$. Su repartición, sin embargo, no siempre es continua y a veces es necesario desplazarse decenas de kilómetros que separan un manchón de otro, a pesar de que el ambiente general no presenta mayores variaciones.

En los alrededores inmediatos del balneario de Lourdes, S.L.P. (aproximadamente a $21^{\circ} 46^{\prime} \mathrm{N}$ y $100^{\circ} 38^{\prime} \mathrm{W}$ ), el matorral dominado por "mariola" cubre en forma total las laderas de los cerros circundantes, algunas de inclinación superior a $45^{\circ}$, en una superficie de muchos kilómetros cuadrados. En este sitio la planta es ubicua y no desprecia habitats francamente ruderales, en los que también llega a abundar.

De Lourdes hacia el norte y el noroeste la especie se extiende sólo a lo largo del cauce del río Santa María y de algunos de sus afluentes; hacia el este (rumbo a San José Albuquerque), hacia el sur (rumbo a Tierra Nueva) y hacia el suroeste (rumbo a Santo 
Acta Botánica Mexicana (1998), 43:57-66

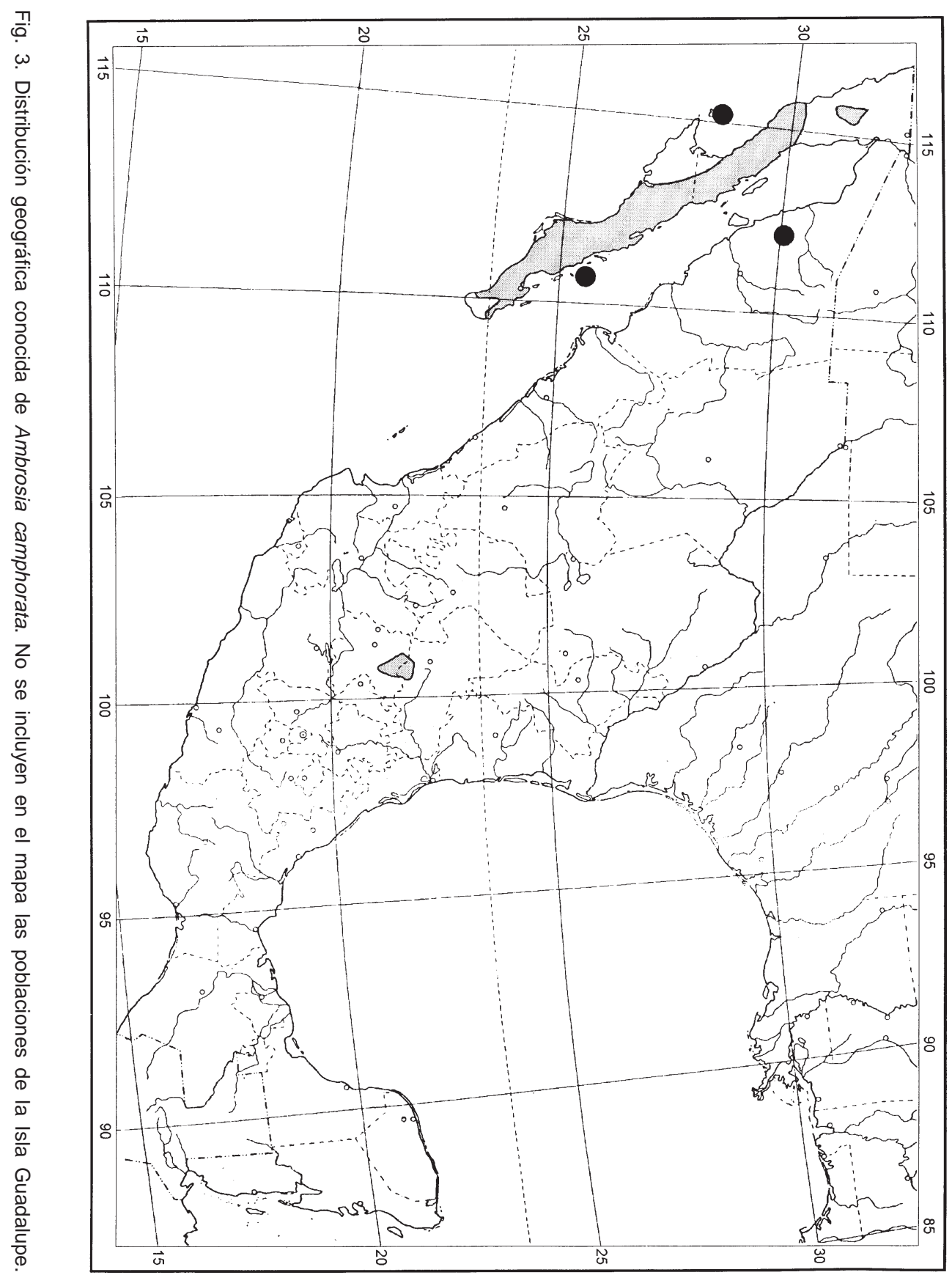

62 
Rzedowski y G. Calderón de Rzedowski: Dos Especies Adventicias de Ambrosia

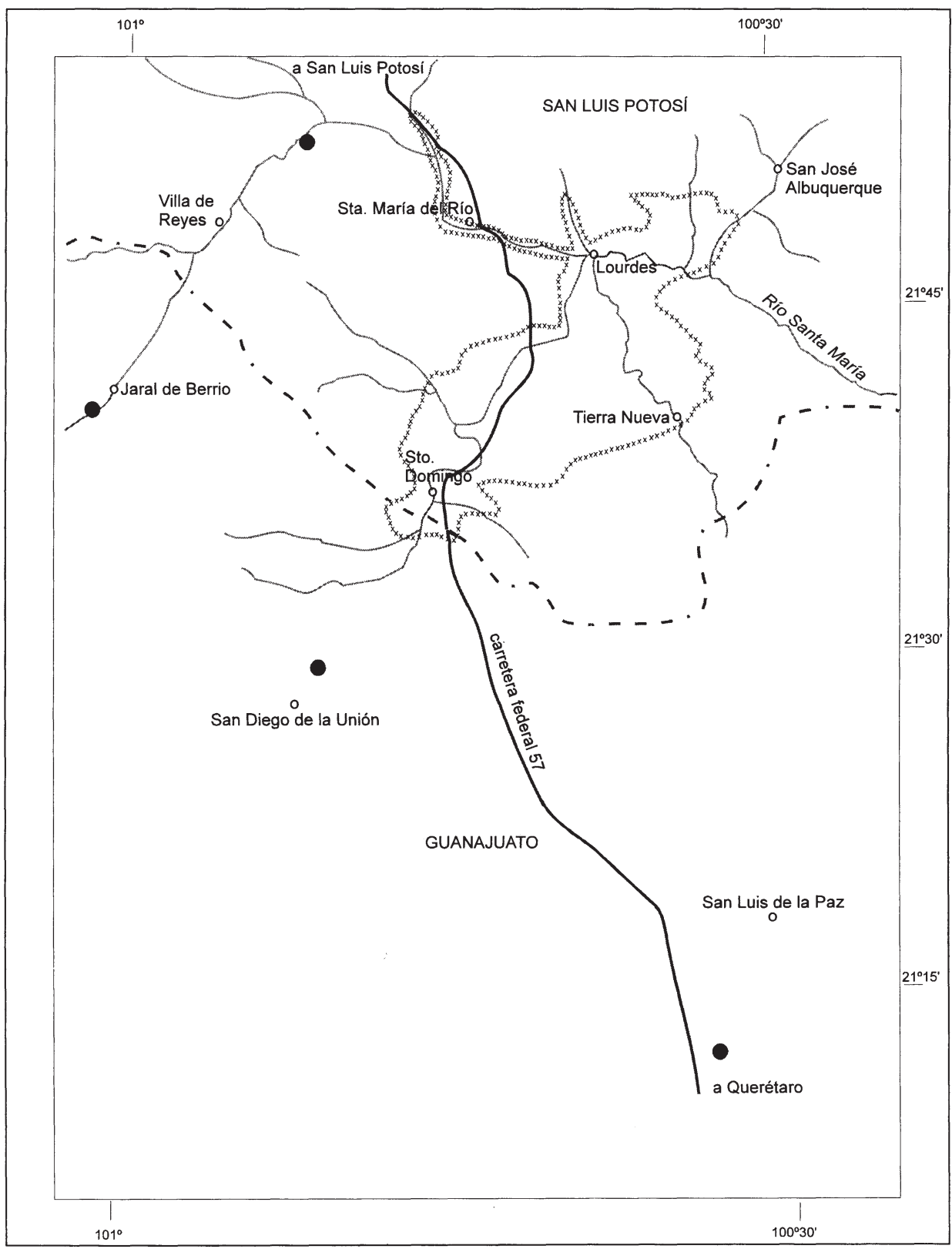

Fig. 4. Distribución geográfica conocida de Ambrosia camphorata en San Luis Potosí y Guanajuato. El área circunscrita por la línea de $x$ es el de una gran concentración de poblaciones. 
Domingo) se observan grandes islas de matorral de $A$. camphorata, pero cada vez más limitadas a terrenos coluviales poco inclinados, ubicados en la base de las laderas. En el estado de Guanajuato, los manchones se tornan definitivamente espaciados y de tamaño reducido y al parecer totalmente restringidos a pendientes suaves afectadas por fuerte disturbio.

En casi todos los sitios $A$. camphorata forma parte del matorral xerófilo y de manera muy notable evade las áreas dominadas por el pastizal.

El hecho de que ambas especies tengan nombres locales bastante bien establecidos en la comarca y además se empleen con cierta frecuencia en la medicina popular indica que estas plantas con toda seguridad han llegado al centro de México antes del presente siglo. Por consiguiente, el descubrimiento tan tardío de la existencia de $A$. camphorata en San Luis Potosí y en Guanajuato obedece más bien a la circunstancia de que la zona no ha sido suficientemente explorada en fechas previas.

Lo anterior se confirma asimismo con la información que se recabó de varios habitantes de mayor edad y conocedores de las plantas de la región, quienes no consideran a ninguna de estas especies de Ambrosia como elementos adventicios de aparición reciente.

El nombre de "mariola", sin embargo, corresponde a Parthenium incanum H.B.K. a todo lo largo de la zona árida chihuahuense y es muy factible que fuera aplicado con posterioridad a $A$. camphorata en virtud de las semejanzas entre ambos arbustos.

En este mismo contexto cabe enfatizar el hecho de que la región árida sonorense indudablemente constituye el centro de diversidad y de probable origen del género Ambrosia (Payne, 1964, 1966). Por consiguiente, A. camphorata y A. cordifolia deben haber arribado al centro de México, procedentes del noroeste. A su vez es importante señalar que los autores no tienen conocimiento de la existencia de otros vegetales superiores que presenten una disyunción similar a la registrada para estas dos plantas.

Dada la distribución análogamente discontinua de ambas, procedería indagar acerca de la época y la forma del movimiento migratorio que las desplazó a distancia tan grande.

A título de hipótesis puede pensarse que el siglo XIX podía haber sido el más propicio y que el viaje puede haberse efectuado en la piel de ganado enviado del norte de Sonora (donde coexisten las dos plantas) al sur de San Luis Potosí.

Desde luego debe considerarse asimismo la posibilidad de que los propágulos de estas plantas llegaron al centro de México transportados por algunas aves o murciélagos migratorios. Tal travesía, sin embargo, no parece muy probable, ya que entonces cabría esperar la presencia de otras xerófitas con disyunción similar a la registrada para las dos especies en cuestión, hecho que aparentemente no tiene lugar. Ya Shreve (1942) puso de manifiesto la notable independencia de las floras de la zonas áridas sonorense y chihuahuense, que en realidad sólo tienen en común un contado número de elementos de amplia distribución.

A continuación se incluye una lista de los ejemplares de herbario procedentes del centro de México que se tuvieron a la vista.

\section{Ambrosia camphorata (Greene) Payne}

San Luis Potosí: cerca de La Turicata, próxima a Pardo, municipio de Villa de Reyes, J. Rzedowski 53580 (IEB); El Toro, cerca de Ojo Caliente, municipio de Santa María del 
Río, J. Rzedowski 53560 (IEB); Santa María del Río, municipio de Santa María del Río, S. del Amo 12 (MEXU); Las Adjuntas, cerca del balneario de Lourdes, municipio de Santa María del Río, J. Rzedowski 53561 (IEB); $10 \mathrm{~km}$ al W de San José Albuquerque, municipio de Santa María del Río, J. Rzedowski 8457 (ENCB); 5 km al NNW de Tierra Nueva, municipio de Tierra Nueva, J. Rzedowski 10793 (ENCB); 2 km al N de Tierra Nueva, municipio de Tierra Nueva, J. Rzedowski 53563 (IEB); Mex 57 km marker 354-355 S of road, municipio de Tierra Nueva, $R$. Scora 2584 (MEXU).

Guanajuato: $2 \mathrm{~km}$ al S de Jaral de Berrio, municipio de San Felipe, J. Rzedowski 53559 (IEB); 4 km al E de San Diego de la Unión, sobre el camino a La Jaula, municipio de San Diego de la Unión, J. Rzedowski 52073, (IEB); 55 mi S of San Luis Potosi traffic circle, municipio de San Diego de la Unión, D. Dunn et al. 20563 (ENCB); Las Beatas, municipio de San Luis de la Paz, R. Santos 107 (IEB).

\section{Ambrosia cordifolia (A. Gray) Payne}

San Luis Potosí: Las Adjuntas, cerca del balneario de Lourdes, municipio de Santa María del Río, J. Rzedowski 53562 (IEB); cerca de Río Higueras, municipio de Santa María del Río, J. Rzedowski 53579 (IEB); 5 km al NW de Tierra Nueva, municipio de Tierra Nueva, J. Rzedowski 10785 (ENCB); 5 km al NNW de Tierra Nueva, municipio de Tierra Nueva, J. Rzedowski 10792 (ENCB); 2 km al N de Tierra Nueva, municipio de Tierra Nueva, J. Rzedowski 53564 (IEB); $15 \mathrm{~km}$ al S de Rioverde, sobre el camino a San Ciro, municipio de Rioverde, J. Rzedowski 8625 (ENCB); municipio de San Ciro, H. Puig 3656 (ENCB).

Guanajuato: La Cieneguilla, municipio de San Luis de la Paz, M. J. Angulo y $L$. Monroy 15 (MEXU); aprox. $10 \mathrm{~km}$ al NE de Doctor Mora, sobre el camino a Victoria, municipio de Victoria, J. Rzedowski 9511 (ENCB, MEXU); Mesa Prieta, municipio de Victoria, R. Santillán 535 (ENCB, IEB); cerca de la mina La Aurora, municipio de Xichú, J. Rzedowski 25540 (IEB); El Banco, 8 km al suroeste de Atarjea, municipio de Atarjea, E. Ventura y E. López 6315 (IEB); 17 km al E de San José Iturbide, municipio de Tierra Blanca, F. Guevara 213 (ENCB).

Querétaro: 2 km al NW de Concá, municipio de Arroyo Seco, J. Rzedowski 25607 (ENCB); cerca de Concá, municipio de Arroyo Seco, J. Rzedowski 42614 (ENCB, IEB); 4 km río abajo del Puente Concá, Río Santa María, municipio de Arroyo Seco, E. Carranza 2328 (IEB); ladera norte y noreste del Cerro Picacho, municipio de Peñamiller, S. Zamudio 2294 (IEB); ladera oriental del Cerro de la Tembladera, $6 \mathrm{~km}$ al $\mathrm{N}$ de Peña Blanca, municipio de Peñamiller, S. Zamudio 3439 (IEB); 33 km al N de Vizarrón, sobre la carretera Jalpan - San Juan del Río, municipio de Peñamiller, S. D. Koch y P. A. Fryxell 7955 (ENCB, MEXU); El Plátano, $30 \mathrm{~km}$ al NE de San Joaquín, municipio de San Joaquín, R. Fernández 4864 (ENCB); camino a S.L.P., municipio de Querétaro, E. Argüelles 1335 (ENCB, MEXU); camino arriba de La Cañada, km 5/6, municipio de El Marqués, E. Argüelles 2731 (IEB, MEXU); camino arriba de La Cañada (Heidi's project), municipio de El Marqués, E. Argüelles 2326 (IEB, MEXU); El Patol, municipio de Tolimán, S. Zamudio 2944 (IEB); 5 km al N de Tolimán, municipio de Tolimán, S. Zamudio 2579 (IEB); $6 \mathrm{~km}$ al NE de Bernal, sobre la carretera a Tolimán, municipio de Tolimán, J. Rzedowski 31596 (MEXU). 


\section{AGRADECIMIENTO}

La confección de los mapas es obra del Biól. Gustavo Barajas.

\section{LITERATURA CITADA}

Blake, S. F. 1926. Asteraceae. In: Standley, P. C. Trees and shrubs of Mexico. Contr. U. S. Nat. Herb. 23: $1401-1641$.

Gentry, H. S. 1942. Rio Mayo plants. A study of the flora and vegetation of the valley of the Rio Mayo, Sonora. Carnegie Inst. Wash. Publ. 527: 1-328.

Hastings, J. R., R. M. Turner y D. K. Warren. 1972. An atlas of some plant distributions in the Sonoran Desert. The University of Arizona. Institute of Athmospheric Physics. Technical Reports on the Meteorology of Arid Regions. No. 21. Tucson, Arizona. 255 pp.

Moran, R. 1996. The flora of Guadalupe Island, Mexico. Mem. Calif. Acad. Sci. 19: 1-190.

Payne, W. W. 1964. A re-evaluation of the genus Ambrosia (Compositae). Journ. Arnold Arbor. 45: 401-430.

Payne, W. W. 1966. Notes on the ragweeds of South America with the description of two new species: Ambrosia pannosa and A. parvifolia (Compositae). Brittonia 18: 28-37.

Rydberg, P. A. 1922. Ambrosiaceae. North Amer. FI. 33: 3-44.

Rzedowski, J. 1961. Vegetación del estado de San Luis Potosí. Tesis. Facultad de Ciencias. Universidad Nacional Autónoma de México. México, D.F. 228 pp.

Rzedowski, J., G. Calderón de Rzedowski y R. Galván. 1996. Nota sobre la vegetación y la flora del noreste del estado de Guanajuato. Flora del Bajío y de Regiones Adyacentes. Fascículo complementario XIV. Instituto de Ecología. Pátzcuaro, Mich. 22 pp.

Shreve, F. 1942. The desert vegetation of North America. Bot. Rev. 8: 195-246.

Shreve, F. 1951. Vegetation of the Sonoran Desert. Carnegie Inst. Wash. Publ. 591: 1-186.

Zamudio Ruiz, S. 1984. La vegetación de la cuenca del Río Estórax en el estado de Querétaro y sus relaciones fitogeográficas. Tesis. Facultad de Ciencias. Universidad Nacional Autónoma de México. México, D.F. 275 pp. 